\title{
Biochemical Markers of Cardiac Risk and Their Relation to Spirometric Indices of Airway Obstruction Among COPD Patients
}

\author{
Sudeb Mukherjee ${ }^{1}$, Debojyoti Bhattacharya ${ }^{2}$, Kaushik Manna ${ }^{3}$ \\ ${ }^{1}$ Department of Cardiology, Institute of Cardiovascular Science, Institute of Post Graduate Medical Education and Research, Kolkata, India \\ ${ }^{2}$ Department of Biochemistry, Calcutta National Medical College \& Hospital, Kolkata, India \\ ${ }^{3}$ Department of Cardiology, Calcutta National Medical College \& Hospital, Kolkata, India
}

Email address:

drsumukherjee@gmail.com (S. Mukherjee)

\section{To cite this article:}

Sudeb Mukherjee, Debojyoti Bhattacharya, Kaushik Manna. Biochemical Markers of Cardiac Risk and Their Relation to Spirometric Indices of Airway Obstruction Among COPD Patients. American Journal of Internal Medicine. Vol. 5, No. 5, 2017, pp. 95-104. doi: 10.11648/j.ajim.20170505.17

Received: May 19, 2017; Accepted: June 5, 2017; Published: October 24, 2017

\begin{abstract}
Monitoring of the cardiac status of COPD (Chronic Obstructive Pulmonary Disease) and comparing them with their respiratory indices thought to be predictive markers of the general health of these patients and be indicative, even prognostic, of any impending deterioration. This study was destined to evaluate various aspect of serum prognostic factors in COPD patients. This study was conducted over a period of six months. During this time biochemical markers of 43 (34 male and 9 female) patients suffering from COPD were collected and compared them with those of 40 healthy case control subjects. The Methodology and Exclusion Criteria were strictly adhered to, and based on the statistical analysis shown below the final results were arrived at, which have been discussed at length below. The relation between smoking and COPD found to be controversial, but smoking none the less has a detrimental effect on the respiratory indices and thus, cessation of smoking would definitely benefit these patients, although the extent of the benefit is a matter of debate and further study. As for the individual markers, serum CRP and plasma homocysteine are definitely important as prognostic markers, as well as the indicators of the present stage of the disease. Serum Lipoprotein (a) was not found to be very effective indicator in this study. Homocysteine as well as CRP but not lipoprotein (a), as cardiac indices is inversely related to the respiratory indices of a COPD patient. Measurement of these parameter might help in prognostic evaluation in those patients.
\end{abstract}

Keywords: Chronic Obstructive Pulmonary Disease (COPD), Homocyteine, C - Reactive Protein (CRP), Lipoprotein (a)

\section{Introduction}

Chronic Obstructive Pulmonary Disease (COPD) is a spectrum of five primary diseases of the lung which are classified as obstructive or airway diseases, as they have the following feature in common $[1,2]$. They cause an increase in airway resistance. The resistance is caused due to an obstruction, either partial or complete, at any level of the airway, starting from the trachea, larger bronchi to the terminal and respiratory bronchioles.

The five primary diseases, however, in their own prototypical forms, have their own distinctive anatomic and clinical features which help us to differentiate between them, and thereby aid therapy as well as management of the cases.
These diseases are:

Chronic Bronchitis, Bronchiectasis, Asthma, Emphysema, Small airway disease $[3,4]$.

Clinically however, a separate classification is used, depending on the extent up to which the obstruction can be corrected, namely reversible and irreversible obstructive disorders. Based on this, it can be classified as follows- 1) Reversible obstructive disease: Asthma 2) Irreversible obstructive disease: Chronic Bronchitis and Emphysema [5].

Clinically, the cases of irreversible airway obstruction are classified as COPD, rather than fully adhering to the true pathological definition of the entity.

Death in patients of COPD is due to several causes that include- Respiratory acidosis and coma, Right sided heart 
failure, Massive collapse of lungs secondary to pneumothorax [6].

COPD is one of the major diseases associated with a high mortality rate. The mortality associated with COPD is so high that the WHO has predicted that that by the year 2020 , COPD will rise from its current ranking as the $12^{\text {th }}$ most prevalent disease worldwide to the $5^{\text {th }}$, and from the $6^{\text {th }}$ most common cause of death to the $3^{\text {rd }}$. Amongst the reasons for the dramatic increase in COPD prevalence, is reduced mortality from causes such as cardiovascular diseases in the developed countries, along with a marked increase in cigarette smoking and environmental pollution in the developing countries [7].

Monitoring of the cardiac status of these subjects and comparing them with their respiratory indices, should therefore, theoretically, be predictive markers of the general health of these patients and be indicative, even prognostic, of any impending deterioration.

Very little work has been conducted in developed countries on the biochemical factors of cardiac risk like homocysteine, lipoprotein a, or systemic inflammatory markers like serum $\mathrm{C}$ reactive Protein (sCRP) in relation to the spirometric indices of COPD, which are used to measure the severity of the disease. Further such reports are likely to vary in a developing country like India, owing to the variations in lifestyle as well as risk factors in morbidity and mortality.

\section{Aims \& Objectives}

1) To test the hypothesis that biochemical markers of cardiac risk like Lipoprotein (a) [Lp (a)], total plasma homocysteine as well as systemic inflammatory markers like serum CRP will be elevated in patients of COPD, as compared to healthy controls.

2) To realize whether the changes in the biochemical markers mentioned above are related to, or independent of the respiratory indices of the patients, and to evaluate the relationship, if any.

3) To assess whether the changes in the biochemical markers are a true reflection of the disease severity and progression.

4) To find out, whether the differences in lifestyle patterns, altered morbidity and mortality scenarios in a developing country like India, have any impact on the changes in the above mentioned biochemical markers.

\section{Materials and Methods}

This is a randomized, single centre, case control study conducted by involving 43 subjects (based on availability of patients at the time of conducting the study), for a period of two months. It involved COPD patients, who had visited the out-patient department. The diagnosis of COPD was made after general and systemic examination, followed by proper radiological analysis and assessment of pulmonary functions, by performing Pulmonary Function Tests (PFTs). Patients with a known history of COPD were also included in this study.

\subsection{Criterion of Inclusion}

a) A definite diagnosis of COPD stated in the patient's medical notes

b) A post bronchodilator $\mathrm{FEV}_{1} /$ forced vital capacity (FVC) ratio of less than $70 \%$.

\subsection{Exclusion Criteria}

(1) Hemoptysis of unknown origin.

(2) Pneumothorax.

(3) Unstable cardiovascular status.

(4) Recent myocardial infarction or pulmonary embolism.

(5) Abdominal or cerebral aneurysms.

(6) Recent eye surgery.

(7) Presence of an acute disease process that might interfere with test performance.

(8) Recent surgery of thorax or abdomen.

(9) Acute infection.

(10)Inflammatory diseases such as rheumatoid arthritis.

(11)History of kidney disease.

\subsection{Collection of Samples and Analysis}

The sample to be assessed was a non fasting venous blood sample. About $5 \mathrm{ml}$ of venous blood was collected aseptically from the ante-cubital vein of the arms of the subjects and were analysed for various biochemical markers. The procedure followed was as follows:

(1) The sample was centrifuged at $3000 \mathrm{rpm}$ for 5 minutes and the supernatant serum is collected by means of a micropipette and stored at $-20^{\circ} \mathrm{C}$.

(2) The cholesterol levels of the subject were first evaluated. If the levels exceeded the normal values (mentioned below), the sample was rejected, as in a subject of hypertriglyceridemia, the levels of $\mathrm{Lp}$ (a) would be elevated at the onset, and would therefore result in a false positive result.

(3) Standards referred to, during the analysis were (as per NCEP-ATP-III):

Total cholesterol: $150-200 \mathrm{mg} / \mathrm{dl}$

HDL fraction: Male: $30-60 \mathrm{mg} / \mathrm{dl}$ Female: $35-75 \mathrm{mg} / \mathrm{dl}$

LDL Fraction: $20-29$ year old: $60-150 \mathrm{mg} / \mathrm{dl}$ 30-39 year old: $80-175 \mathrm{mg} / \mathrm{dl}$ 40-49 year old: $90-200 \mathrm{mg} / \mathrm{dl}$

(4) Since all the patients were past the $4^{\text {th }}$ decade of life, COPD being more prevalent in this age group, the accepted level of LDL cholesterol was $20-200 \mathrm{mg} / \mathrm{dl}$.

(5) Following this, the serum was used to assess the various biochemical markers, namely

(i) Serum CRP: measured by immunoturbidimetry

(ii) Lipoprotein (a): measured by immunoturbidimetry

(iii) Total plasma homocysteine: measured by ELISA.

(6) The results were documented along with - name, age, gender of subject, any history of smoking, date of assessment. 


\subsection{Measurement of Spirometric Variables}

A handheld turbine spirometer was used for the measurement of the spirometric variables of the patient. The spirometer was set at an appropriate calibration and did not require re-calibration. The procedure was done in the following steps:

(1) The subject is asked to respire normally for 2-3 times. Then he is asked to inhale maximally, and then exhale with as much force as they can and as long as they can. From here Forced Vital Capacity (FVC) was collected and the part of the Forced Vital Capacity exhaled in 1 second, called Forced Expiratory Volume in 1 second $\left(\mathrm{FEV}_{1}\right)$. Both these data are tabulated, and the ratio of $\mathrm{FEV}_{1} / \mathrm{FVC}$ is calculated.

(2) Following this, the subject is given two puffs of salbumatol (100 micrograms) and are made to repeat the spirometry. This is done to eliminate any reversible component of airway obstruction, such as asthma, that is sensitive to bronchodilator therapy. Thus in an individual with reversible obstruction, the $\mathrm{FEV}_{1} / \mathrm{FVC}$ ratio will increase, as compared to a person with irreversible obstruction in whom it will remain unchanged. If the post bronchodilator $\mathrm{FEV}_{1} / \mathrm{FVC}$ ratio is less than $70 \%$, the subject was chosen for the study.

With the help of the above materials and methods the study has been carried out in the stipulated time period. Case study forms that contain the information necessary for our study have been filled up in order. Based on the information available on from these forms and the data collected, a detailed analysis, shown below, had been carried out. The analysis yielded some significant results (supported by statistical data) and have been tabulated below.

\section{Observations and Results}

In this study, 43 COPD patients was selected at random and 40 relatively healthy subjects as controls, on whom the analysis was conducted. As elaborated in the methodology, the diagnosis of COPD was made after General examination, Biochemical analysis and Spirometry, subject to the fulfillment of our inclusion criterion.

The following significant results were obtained:

Age: This study revealed the following relation with respect to age of the subject (Table 1)

Table 1. Total number of subjects $(n)=43$ subjects.

\begin{tabular}{lllll}
\hline \multirow{2}{*}{ Age of subject } & $\begin{array}{l}\mathbf{4 0 - 5 0} \\
\text { years }\end{array}$ & $\begin{array}{l}\mathbf{5 0 - 6 0} \\
\text { years }\end{array}$ & $\begin{array}{l}\mathbf{6 0 - 7 0} \\
\text { years }\end{array}$ & $\begin{array}{l}\mathbf{7 0 - 8 0} \\
\text { years }\end{array}$ \\
\hline Number of subjects & 05 & 16 & 12 & 10 \\
Percentage of subjects & $11.67 \%$ & $37.20 \%$ & $27.90 \%$ & $23.25 \%$ \\
\hline
\end{tabular}

Sex: Age-Sex Distribution Pattern of this study has been tabulated in Table 2 and Fig.-1.

Table 2. Age-Sex Distribution of the Subjects.

\begin{tabular}{lll}
\hline Age group & Male & Female \\
\hline $40-50$ & 3 & 2 \\
$50-60$ & 14 & 2 \\
$60-70$ & 11 & 1 \\
$70-80$ & 6 & 4 \\
\hline
\end{tabular}

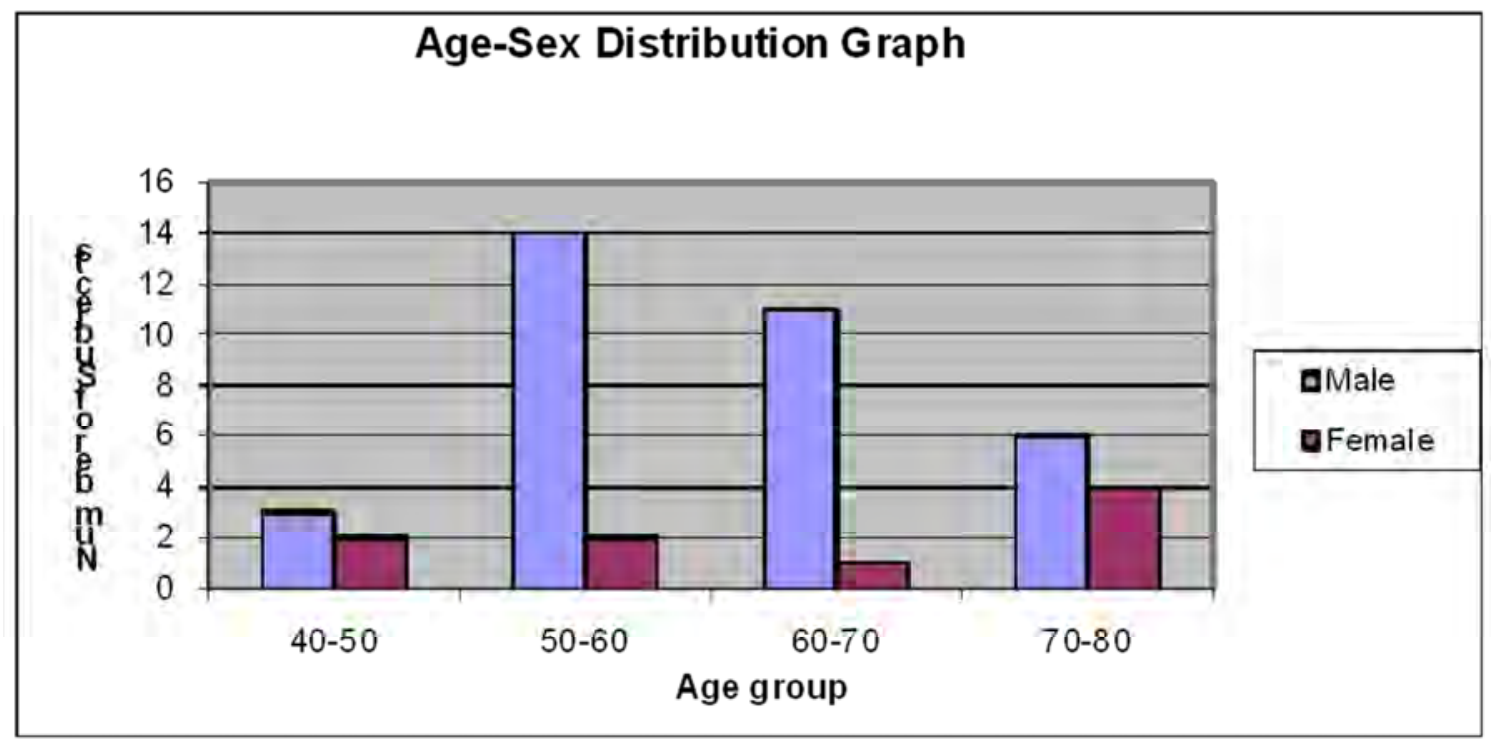

Figure 1. Showing distribution of studied population.

Analyzing the Table 3 with Karl Pearson's Correlation Coefficient the following results was found: $\mathrm{r}_{1}$ (between $\mathrm{FEV}_{1} / \mathrm{FVC}$ and serum CRP) $=-0.99, \mathrm{r}_{2}$ (between $\mathrm{FEV}_{1} / \mathrm{FVC}$ and plasma homocysteine) $=-0.99, \mathrm{r}_{3}$ (between $\mathrm{FEV}_{1} / \mathrm{FVC}$ and lipoprotein (a) ) $=-0.043$, and $P$ value: $<0.05$. This was done to evaluate whether association was more in female or not. The same association was shown in Table 4 in male patients. Analyzing this Table 4 with Karl Pearson's Correlation Coefficient, the following results was found: $r_{1}$ (between $\mathrm{FEV}_{1} / \mathrm{FVC}$ and serum CRP) $=-0.99, \mathrm{r}_{2}$ (between $\mathrm{FEV}_{1} / \mathrm{FVC}$ and plasma homocysteine) $=-0.99, \mathrm{r}_{3}$ (between $\mathrm{FEV}_{1} / \mathrm{FVC}$ and lipoprotein $\left.(\mathrm{a})\right)=-0.159$ and $\mathrm{P}$ value: $<0.05$ 
Table 3. Total number of female subjects $(n)=9$.

\begin{tabular}{llll}
\hline FEV $_{\mathbf{1}} /$ FVC ratio (in \%) & Serum CRP $(\mathbf{m g} / \mathbf{l})$ & Total plasma homocysteine (micromoles/L) & Lipoprotein (a) (mg/d) \\
\hline 54.5 & 4.87 & 10.70 & 12.5 \\
58.53 & 4.38 & 9.96 & 11 \\
61.53 & 4.02 & 9.42 & 13.1 \\
58.9 & 4.34 & 9.89 & 15 \\
58.18 & 4.42 & 10.03 & 10.3 \\
66.2 & 3.46 & 8.58 & 12.2 \\
50 & 5.4 & 11.5 & 12.8 \\
61.76 & 3.99 & 9.38 & 31.3 \\
69.1 & 3.12 & 8.06 & 7.2 \\
\hline
\end{tabular}

Table 4. Total number of Male Subjects $(n)=34$.

\begin{tabular}{|c|c|c|c|}
\hline$F E V_{1} / F V C$ ratio (in \%) & Serum CRP ( mg/l) & Plasma homocysteine (micromoles/L) & Lipoprotein (a) (mg/dl) \\
\hline 68.5 & 3.19 & 8.17 & 14 \\
\hline 66.66 & 3.4 & 8.48 & 14.2 \\
\hline 56.9 & 4.58 & 10.26 & 14.4 \\
\hline 52.41 & 5.12 & 11.07 & 7.2 \\
\hline 60.22 & 4.18 & 9.66 & 9.3 \\
\hline 62.33 & 3.93 & 9.28 & 6 \\
\hline 33.3 & 7.41 & 14.5 & 12 \\
\hline 58.04 & 4.44 & 10.05 & 7.1 \\
\hline 56.52 & 4.63 & 10.33 & 6.3 \\
\hline 58.62 & 4.37 & 9.94 & 8 \\
\hline 40.7 & 6.52 & 13.17 & 13 \\
\hline 61.24 & 4.06 & 9.48 & 14.1 \\
\hline 65.97 & 3.49 & 8.63 & 13.05 \\
\hline 59.73 & 4.24 & 9.75 & 11.03 \\
\hline 66.66 & 3.41 & 8.5 & 15.2 \\
\hline 61.04 & 4.08 & 9.51 & 9.2 \\
\hline 45.93 & 5.89 & 12.23 & 7.88 \\
\hline 38.6 & 6.76 & 13.55 & 8.79 \\
\hline 60.95 & 4.1 & 9.53 & 8.62 \\
\hline 64.44 & 3.68 & 8.9 & 5.3 \\
\hline 64.1 & 3.72 & 8.97 & 9.19 \\
\hline 34.4 & 7.27 & 14.31 & 12.07 \\
\hline 63.63 & 3.77 & 9.05 & 11.36 \\
\hline 64.95 & 3.62 & 8.81 & 7.2 \\
\hline 66.97 & 3.4 & 8.44 & 7.35 \\
\hline 69.39 & 3.08 & 8.01 & 7.51 \\
\hline 53.1 & 5.04 & 10.94 & 10 \\
\hline 49.7 & 5.44 & 11.55 & 14.7 \\
\hline 58.53 & 4.36 & 10 & 15 \\
\hline 39.5 & 6.65 & 13.38 & 14.6 \\
\hline 56.97 & 4.57 & 10.24 & 8.3 \\
\hline 58.15 & 4.43 & 10.03 & 6.5 \\
\hline 56.21 & 4.66 & 10.4 & 6.43 \\
\hline 59.28 & 4.29 & 9.83 & 6.34 \\
\hline
\end{tabular}

Inter-individual variance among the biochemical markers in the female and male patients has been shown in Figure 2 and Figure 3 respectively. 


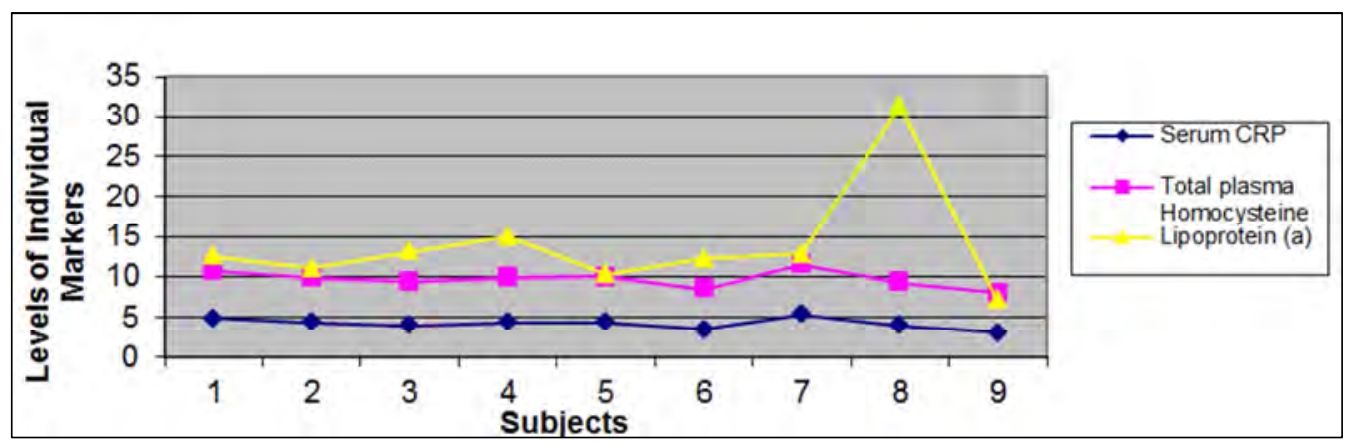

Figure 2. Graph showing the Inter-individual variance among the biochemical markers in the female subjects $(n=9)$.

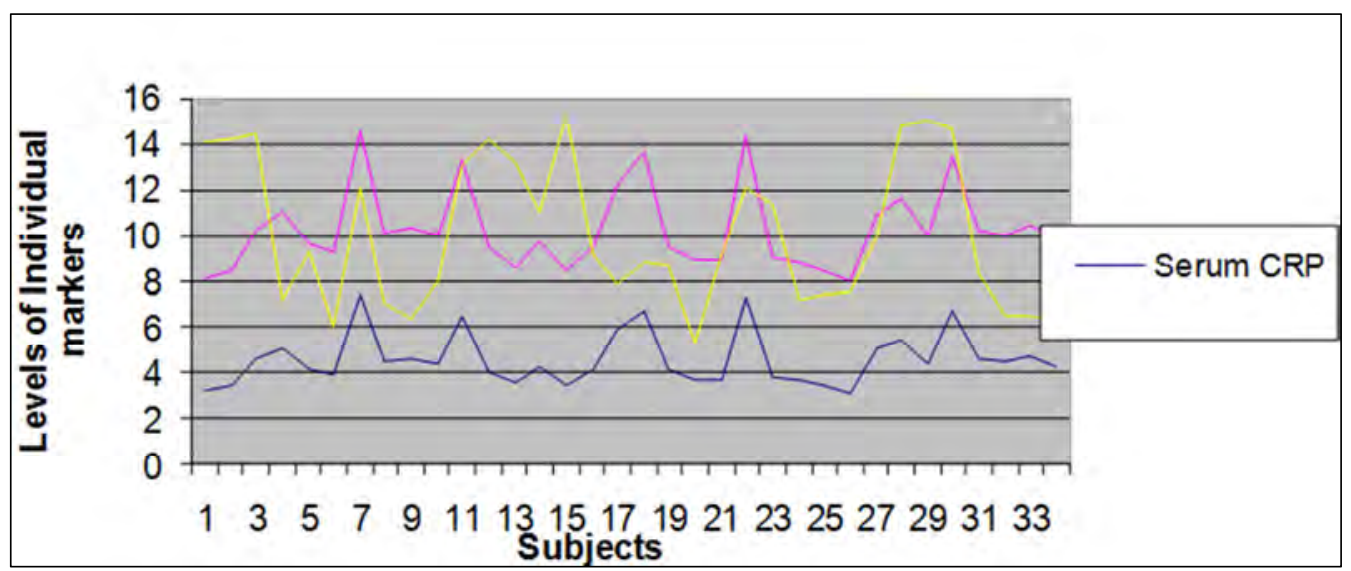

Figure 3. Graph showing the Inter-individual variance among the biochemical markers in the male subjects. $(n=34)$.

History of smoking: The following is an analysis of the $\mathrm{FEV}_{1} / \mathrm{FVC}$ ratio of the subjects who smoked, in comparison to the number of pack- years smoked. 8 subjects failed to provide a definite number of pack years and hence were omitted from this analysis.

Figure 4 depicted inter-individual comparison between number of Pack Years and FEV $/$ FVC Ratio. Karl Pearson's correlation coefficient $\mathrm{r}=+0.033517$. P value: $<0.05$

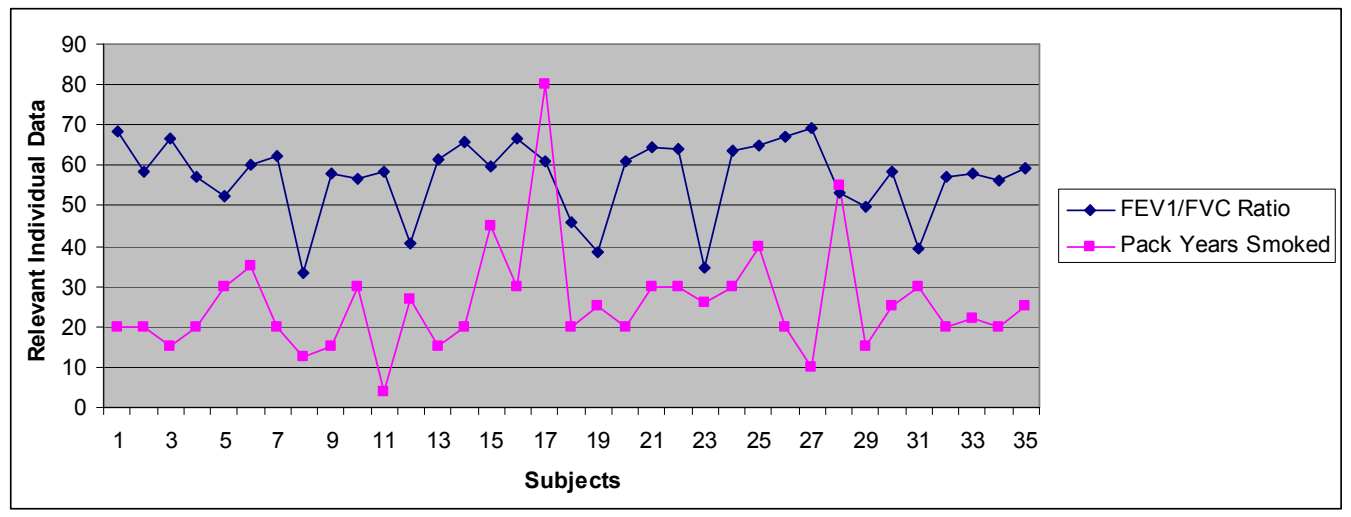

Figure 4. Inter-individual comparison between number of Pack Years and FEV $V_{l} / F V C$ Ratio.

Individual Biochemical Markers: After the above analysis, we analyzed the biochemical markers, serum CRP, Total plasma homocysteine and Lipoprotein (a) individually, noting the inter-relationships with the spirometric indices of respiratory disease and smoking history.

Serum C Reactive Protein:

Table 5 depicts comparison between Serum CRP levels and FEV1/FVC Ratio. Karl Pearson's Correlation Co-efficient $=$ 0.99 , P value: $<0.05$.

Figure 5 showed graphical Comparison between Serum CRP and Number of Pack Years.

Karl Pearson's Co-efficient: -0.033 . P value: $<0.05$ 


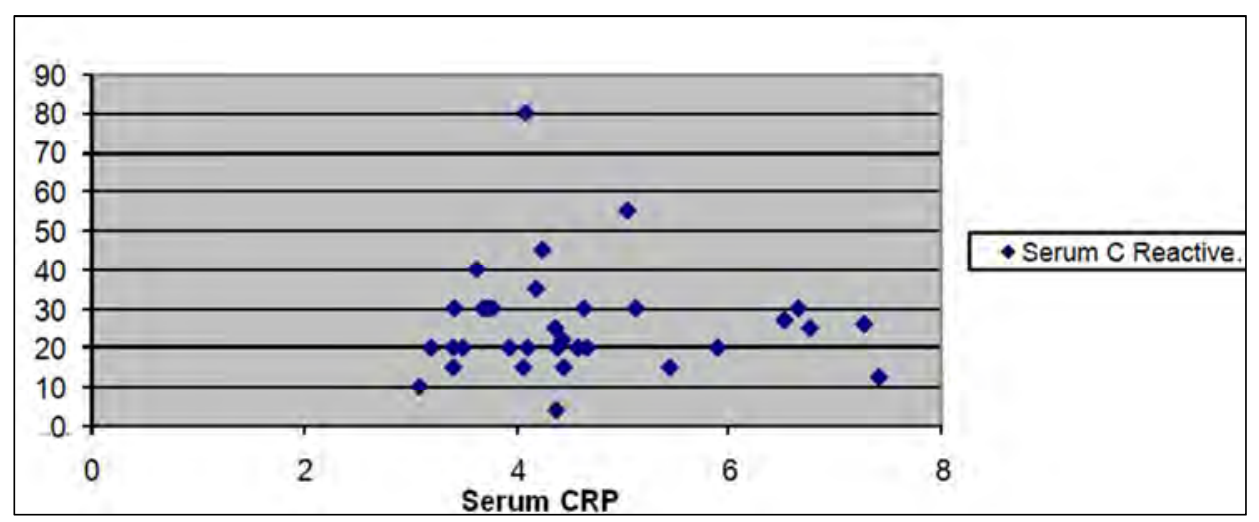

Figure 5. Graphical Comparison between Serum CRP and Number of Pack Years.

Table 5. Comparison between Serum CRP levels and FEV1/FVC Ratio.

\begin{tabular}{|c|c|}
\hline FEV $/$ FVC Ratio (in \%) & Serum C Reactive Protein (in mg/L) \\
\hline 54.5 & 4.87 \\
\hline 68.5 & 3.19 \\
\hline 58.53 & 4.38 \\
\hline 66.66 & 3.4 \\
\hline 61.53 & 4.02 \\
\hline 58.9 & 4.34 \\
\hline 58.18 & 4.42 \\
\hline 56.9 & 4.58 \\
\hline 66.2 & 3.46 \\
\hline 50 & 5.4 \\
\hline 61.76 & 3.99 \\
\hline 52.41 & 5.12 \\
\hline 60.22 & 4.18 \\
\hline 62.33 & 3.93 \\
\hline 33.3 & 7.41 \\
\hline 58.04 & 4.44 \\
\hline 56.52 & 4.63 \\
\hline 58.62 & 4.37 \\
\hline 40.7 & 6.52 \\
\hline 61.24 & 4.06 \\
\hline 65.97 & 3.49 \\
\hline 59.73 & 4.24 \\
\hline 66.66 & 3.41 \\
\hline 61.04 & 4.08 \\
\hline 45.93 & 5.89 \\
\hline 38.6 & 6.76 \\
\hline 60.95 & 4.1 \\
\hline 64.44 & 3.68 \\
\hline 64.1 & 3.72 \\
\hline 34.4 & 7.27 \\
\hline 63.63 & 3.77 \\
\hline 64.95 & 3.62 \\
\hline 66.97 & 3.4 \\
\hline 69.39 & 3.08 \\
\hline 53.1 & 5.04 \\
\hline 49.7 & 5.44 \\
\hline 58.53 & 4.36 \\
\hline 39.5 & 6.65 \\
\hline 69.1 & 3.12 \\
\hline 56.97 & 4.57 \\
\hline 58.15 & 4.43 \\
\hline 56.21 & 4.66 \\
\hline 59.28 & 4.29 \\
\hline
\end{tabular}

Total Plasma Homocysteine:

Figure 6 denotes comparison between Total Plasma Homocysteine and Number of Pack Years. Karl Pearson's Co-efficient (r): 0.99. P value: $<0.05$

And with respect to history of smoking (Table 6) Karl Pearson's correlation co-efficient was 0.030 with P value $<0.05$. 


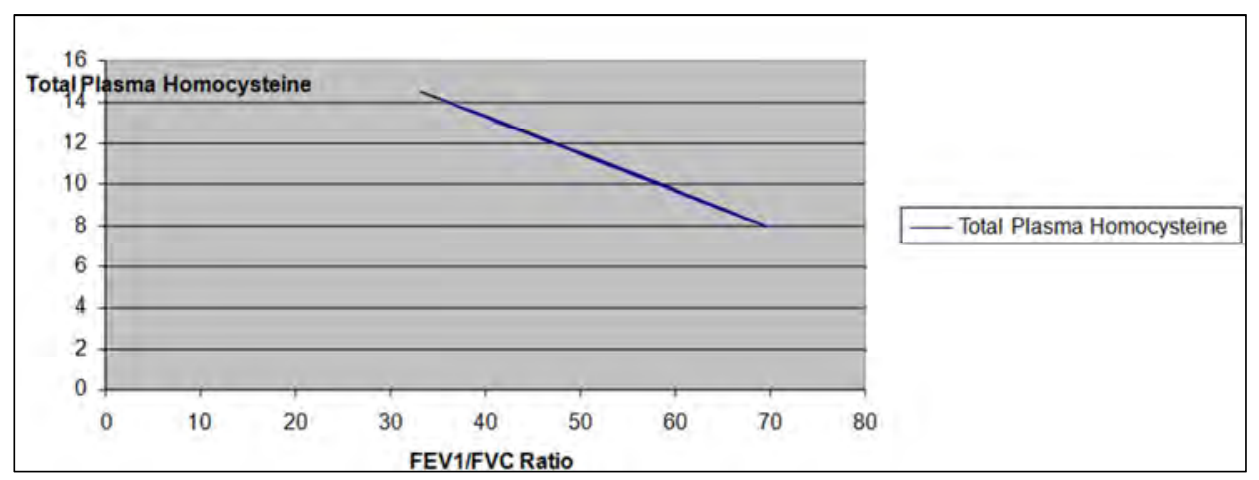

Figure 6. Comparison between Total Plasma Homocysteine and Number of Pack Years.

Table 6. Comparison between Total Plasma Homocysteine and Number of Pack Years.

\begin{tabular}{|c|c|}
\hline $\mathrm{FEV}_{1} / \mathrm{FVC}$ Ratio (in \%) & Total plasma homocysteine (in micromoles/L) \\
\hline 54.5 & 10.7 \\
\hline 68.5 & 8.17 \\
\hline 58.53 & 9.96 \\
\hline 66.66 & 8.48 \\
\hline 61.53 & 9.42 \\
\hline 58.9 & 9.89 \\
\hline 58.18 & 10.03 \\
\hline 56.9 & 10.26 \\
\hline 66.2 & 8.58 \\
\hline 50 & 11.5 \\
\hline 61.76 & 9.38 \\
\hline 52.41 & 11.07 \\
\hline 60.22 & 9.66 \\
\hline 62.33 & 9.28 \\
\hline 33.3 & 14.5 \\
\hline 58.04 & 10.05 \\
\hline 56.52 & 10.33 \\
\hline 58.62 & 9.94 \\
\hline 40.7 & 13.17 \\
\hline 61.24 & 9.48 \\
\hline 65.97 & 8.63 \\
\hline 59.73 & 9.75 \\
\hline 66.66 & 8.5 \\
\hline 61.04 & 9.51 \\
\hline 45.93 & 12.23 \\
\hline 38.6 & 13.55 \\
\hline 60.95 & 9.53 \\
\hline 64.44 & 8.9 \\
\hline 64.1 & 8.97 \\
\hline 34.4 & 14.31 \\
\hline 63.63 & 9.05 \\
\hline 64.95 & 8.81 \\
\hline 66.97 & 8.44 \\
\hline 69.39 & 8.01 \\
\hline 53.1 & 10.94 \\
\hline 49.7 & 11.55 \\
\hline 58.53 & 10 \\
\hline 39.5 & 13.38 \\
\hline 69.1 & 8.06 \\
\hline 56.97 & 10.24 \\
\hline 58.15 & 10.03 \\
\hline 56.21 & 10.4 \\
\hline 59.28 & 9.83 \\
\hline
\end{tabular}

Serum Lipoprotein (a):

Figure 7 and Table 7 showed graphical comparison between Lipoprotein (a) and FEV1/FVC Ratio and with respect to history of smoking respectively. Karl Pearson's Co-efficient: 0.10 and 0.05 in Figure 7 and Table 7 respectively with p value $<$ 0.05 . 
Table 7. Comparison between Serum Lipoprotein (a) and Number of Pack Years.

\begin{tabular}{|c|c|}
\hline Number of Pack Years & Serum Lipoprotein (a) (in $\mathrm{mg} / \mathrm{L}$ ) \\
\hline 20 & 14 \\
\hline 20 & 11 \\
\hline 15 & 14.2 \\
\hline 20 & 14.4 \\
\hline 30 & 7.2 \\
\hline 35 & 9.3 \\
\hline 20 & 6 \\
\hline 12.5 & 12 \\
\hline 15 & 7.1 \\
\hline 30 & 6.3 \\
\hline 4 & 8 \\
\hline 27 & 13 \\
\hline 15 & 14.1 \\
\hline 20 & 13.05 \\
\hline 45 & 11.03 \\
\hline 30 & 15.2 \\
\hline 80 & 9.2 \\
\hline 20 & 7.88 \\
\hline 25 & 8.79 \\
\hline 20 & 8.62 \\
\hline 30 & 5.3 \\
\hline 30 & 9.19 \\
\hline 26 & 12.07 \\
\hline 30 & 11.36 \\
\hline 40 & 7.2 \\
\hline 20 & 7.35 \\
\hline 10 & 7.51 \\
\hline 55 & 10 \\
\hline 15 & 14.7 \\
\hline 25 & 15 \\
\hline 30 & 14.6 \\
\hline 20 & 14 \\
\hline 22 & 11 \\
\hline 20 & 14.2 \\
\hline 25 & 14.4 \\
\hline
\end{tabular}

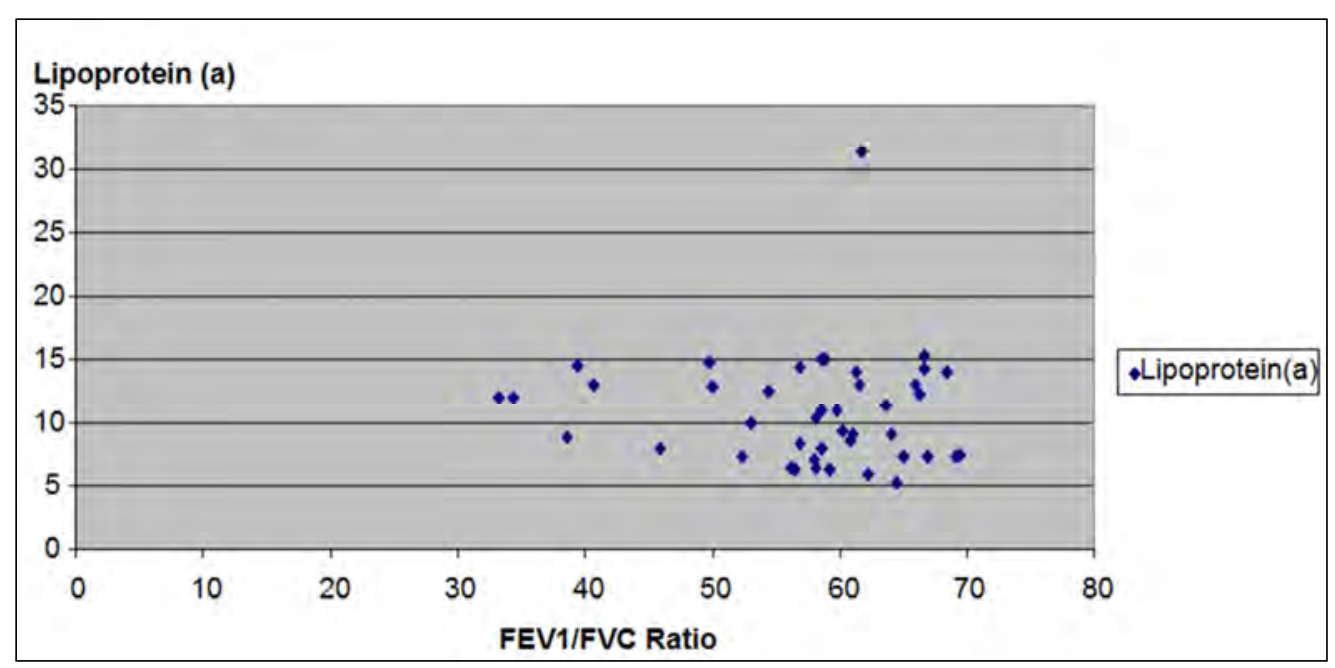

Figure 7. Comparison between Lipoprotein (a) and FEV1/FVC Ratio.

\section{Discussion}

It is a well known fact, that a progressive, obstructive disorder like COPD becomes symptomatic after it exhausts the physiological reserve of the lungs. This is reflected in the fact that the incidence of COPD increases in the $4^{\text {th }}$ decade of life.
In this study, the lowest age group was $40-50$ years (Table 1). The number of subjects rising to $37.20 \%$ in the $50-50$ year category. Thereafter however, the numbers gradually decrease to $27.90 \%$ in the $60-70$ year and to $23.25 \%$ in the $70-80$ year category, the latter being due increased mortality owing to uncontrolled disease progression.

COPD is predominantly a disease affecting the male 
population. In our study (which was randomized) showed same results (Table 2).

The relation between the respiratory indices and smoking habit (in terms of pack years) has been analysed in this study. Out of the 43 subjects, 35 were smokers and were considered for this analysis. The gender bias, if any, was not assessed, because out of the 9 female subjects, 8 were non smokers. This analysis (Figure 4), however, yielded $r=+0.033$, which indicates a positive correlation, rather than the expected negative one. This suggests, that it is not only smoking, but other factors that culminate together to cause disease progression, even in a smoking population. Thus smoking may be an aggravating factor, as well as an initiating factor, and the disease process may progress, albeit at a slower rate, even is the person gives up smoking, in the presence of other predisposing factors [8].

This study revealed serum CRP was elevated in the COPD subjects at a mean of $4.50(3.08-7.41) \mathrm{mg} / \mathrm{L}$ than in the case controls. Compared to the study by Seemungal et al [7], who estimated Serum CRP at $2.05 \mathrm{mg} / \mathrm{l}$, this study revealed a higher value, indicating that altered morbidity and mortality patterns in developing nations do have a bearing on serum CRP levels. A correlation of $r=0.99$ was found with the respiratory indices that shows a strong inverse correlation. Such higher value of CRP was also found in several other studies reported outside India [9, 10].

With smoking history, a weaker, but significant $\mathrm{r}$ value $=$ 0.033 was found, which indicates that smoking does have a detrimental effect on serum CRP values and may have an impact on its progressive rise among COPD patients (Table 5).

The analysis was also done for total plasma homocysteine (Figure 6). Plasma Homocysteine was elevated in the COPD subjects at a mean of $10.15(8.01-14.50)$ micromoles/L than in the controls. A lower mean value was found in this study, which shows that plasma homocysteine is not significantly affected by altered morbidity and mortality conditions of developing nations. In reality, the lifestyle patterns of developed countries, seems to have a greater impact on it. A correlation of $\mathrm{r}=0.99$ was found with the respiratory indices that shows a strong inverse correlation. With smoking a significant $r$ value of 0.030 (Table 6) was found, which indicates that smoking does have a detrimental effect on plasma homocysteine values and may have an impact on its progressive rise among COPD patients. This results is in accordance with several other studies published [11].

Serum lipoprotein (a) was not significantly elevated in the COPD patients. The mean value was found to be 7.785 micromoles/l. A correlation of $r=0.05$ was found with the respiratory indices that shows a weaker inverse correlation. This shows that lipoprotein (a) may have a direct effect in the progression of the disease or may be adjunctive and increase with deteriorating cardiac health as the disease progresses.

With smoking history $r$ value was found to be 0.010 , which indicates that smoking has a weakly detrimental effect on serum lipoprotein (a) values.

Whether COPD has any effect on existing LDL levels is yet to be comprehensively proven. In this study, the subjects were screened, so that they do not have an altered lipid profile. Thus the obtained results based purely on the interaction of lipid profile and smoking history or respiratory indices. It seems, from our results, that COPD has no significant effect on Lipoprotein (a) levels, and the findings may be incidental in nature, hence subject to further study.

\section{Conclusion}

Thus, based on the above findings following facts can be comprehensively stated.

(1) Biochemical marker of Cardiac disease, homocysteine is inversely related to the respiratory indices of a COPD patient.

(2) Similar inverse correlation can be evaluated between markers of systemic inflammation, serum CRP and respiratory indices

(3) The status of Lipoprotein (a) is controversial, and it requires further analysis. In this study however, a weak inverse relationship was obtained.

(4) The biochemical markers assessed have a definite relationship with disease progression, and are not independent of the disease process. Amongst the 3 markers assessed, serum CRP and Plasma homocysteine are equivalently sensitive to the progressing disease process. However, in terms of cardiac health analysis, plasma homocysteine is preferred as it is cardio-specific, compared to serum CRP, which may be elevated in several inflammatory conditions, and thus not a true indicator of cardiac health. Lipoprotein (a) status being controversial, and much weakly related to disease progression as compared to homocysteine, is not a good prognostic marker in this case

(5) Smoking as a major causative factor is another controversial issue, because while this study establish that it does impact all 3 of studied biochemical markers, it has also been shown that smoking does not bear a clear cut inverse relationship with respiratory indices. Further, the ill effects of smoking are not restricted to COPD only, as it may individually cause CHD, or other systemic inflammatory conditions, altering Plasma homocysteine, Lipoprotein (a) and serum CRP. Thus this fact needs further study, to define the exact status of smoking in the causality of COPD.

\section{References}

[1] Parameswaram GI, Murphy TF: Infections in chronic lung diseases. Infect Dis Clin North Am 21: 673, 2007.

[2] Thurlbeck WM, Chronic obstructive pulmonary disease Philadelphia, WB Saunders, 1991.

[3] Hogg JC, Timens W: The pathology of Chronic obstructive pulmonary disease. Ann Rev Path Mech Dis 4: 435, 2009. 
[4] Taraseviceine-Stewart L, Voekel NF: Molecular pathogenesis of emphysema, J Clin Invest 118: 394, 2008.

[5] Miravittles M, Jose Soler Cataluna J, Calle M, Soriano JB. Treatment of COPD by clinical phenotypes. Putting old evidence into clinical practice. Eur Respir J 2012 Oct 11.

[6] Minas M, Verrou-Katsarou I, Mystriduo P, Apostolidou E, Hatzoglou C, Gourgoulianis KI. Two year mortality of patienst with COPD in primary health care: an observational study. Int J Gen Med 2012; 5: 815-22.

[7] Seemungal TA et al, Plasma homocysteine is elevated in COPD patients and is related to COPD severity. Int $\mathrm{J}$ of Chron Obstuct Pulmon Dis 2007; 2(3): 313-21.

[8] Folchini F, Nonato NL, Feofiloff E, D'Almeida V, Nascimento $\mathrm{O}$, Jardim JR, Association of oxidative stress markers and Creactive protein with multidimensional indexes in COPD. Chron Respir Dis 2011; 8(2): 101-8. Epub 2011 Mar 24.
[9] Aksu F, Capan N, Aksu K, Ofluoğlu R, Canbakan S, Yavuz B, Akin KO. C-reactive protein levels are raised in stable chronic obstructive pulmonary disease patients independent of smoking behavior and biomass exposure. J Thorac Dis. 2013; 5: 414-421.

[10] Z. C. Deng, P. Zhao, C. Cao, S. F. Sun, F. Zhao, C. Y. Lu, H. Y. Ma. C-reactive protein as a prognostic marker in chronic obstructive pulmonary disease Exp. Ther. Med., 7 (2014), pp. 443-446.

[11] Beeh, K. M., Watz, H., Puente-Maestu, L., de Teresa, L., Jarreta, D., Caracta, C., Garcia Gil, E., and Magnussen, H. Aclidinium improves exercise endurance, dyspnea, lung hyperinflation, and physical activity in patients with COPD: a randomized, placebo-controlled, crossover trial. BMC Pulm. Med. 2014; 14: 209. 